\title{
Congestion Management Approaches in Deregulated Power System - An Illustrative Approach
}

\author{
Varaprasad Janamala \\ Assistant Professor, Dept. of Electrical Engineering \\ Faculty of Engineering, Christ University, Bangaluru - 560074 \\ varaprasad.janamala@chraistuniversity.in
}

\begin{abstract}
In deregulated power system with competitive electricity market environment, the provision of strategic bidding option to the market participants and its consequences are open up new challenging tasks to the system operator. The market economic efficiency is mainly dependent on transmission system support. The inability of transmission system support to drive market cleared schedule is known as congestion and which is not desirable. The remedial actions to relieve congestion in the transmission system known as congestion relief approaches and are differ in various markets around the world. The objective of this paper is to illustrate some of the technical and non-technical approaches using simple case studies.
\end{abstract}

Keywords: Deregulated power system, competitive electricity market, congestion management

\section{Introduction}

By the competitive electricity market environment with deregulation, the way of power system operation and management have been changed drastically but system security and reliability requirements are still remains same. The provision for market participants to change their bidding option at any trading interval during market settlement process has further created more competitive options. Due to this, the generation schedules and load withdrawals become random in nature and unpredictable. In order to facilitate all types of market operations satisfactorily, the transmission system support is playing a key role. In market terminology, the inability of transmission system to dispatch market equilibrium point is treated as congestion and the corresponding remedial actions to relieve congestion are termed as congestion management (CM) approaches. Real-time transmission congestion can be defined as the operating condition in which there will not be enough transmission capability to implement all the traded transactions simultaneously due to some unexpected contingencies. It may be alleviated by incorporating line capacity constraints in the dispatch and scheduling process. This may involve re-dispatch of generation or load curtailment.

Transmission congestion can alleviate through various ways. A comprehensive literature survey on various congestion relief approaches has been presented in [1]. All these approaches can divide in to two types which are like technical and non-technical. Among the technical solutions we have outaging of congested lines, operation of FACTS devices and operation of transformer tap changers, control of VAr supporting devices etc. Among the non technical solutions we have market based and non-market based methods of CM.

Non-market based methods are those where no form of market mechanism is used to allocate the scarce transmission capacity but use other reasonable criteria. These include sharing of capacity on a pro rata basis where users share in proportion to their requirements, first come first serve and preference for certain types of contracts. The non- 
market based methods for congestion management do not send any signals for investment and have no measure of the value of the congested line. Market based methods are based on market mechanisms and hence give an indication of the value of the scarce resource of transmission capacity. The classification of CM approaches is given Table 1.

Table 1. Classification of Congestion Management Approaches

\begin{tabular}{llll}
\hline \hline \multicolumn{1}{c}{ Technical Methods } & & \multicolumn{2}{c}{ Non-Technical Methods } \\
\cline { 1 - 1 } a) Outaging of congested lines & & Market-based & Non-market based \\
b) Transformer tap changers & & a) Auctioning & a) First come, first serve \\
c) Operation of FACTS devices & & b) Market splitting & b) Pro rata \\
d) Control of Volt/VAr & & c) Counter trading & \\
& supporting devices & d) Redispatching & \\
& e) Load curtailment \\
& f) Nodal pricing \\
\hline
\end{tabular}

According to [2], the above said CM approaches can also classify in to two ways: cost free and non-cost free approaches. Some of these approaches like operation of FACTS devices, tap-changing transformers, capacitor banks or VAr control etc., are best examples for cost free methods since there is no involvement of market participants and hence market economics will remains same as at equilibrium. On other side, like rescheduling and load curtailment or adjustment programs in non-cost free approaches are causes to change market equilibrium and hence changes in market economics. By the adoption of cost free approaches, we can avoid market abuse by participants.

The major objective of this paper is to illustrate some of the technical approaches like operation of FACTS devices, control of Volt/VAr supporting devices and non-technical approaches like redispatch and load curtailment in a simplest way.

\section{Test System and Base Case Solution}

The test system has 6 buses and is divided into two areas. Area-1 has buses 1, 3 and 6 with generator at bus-1. Similarly Area- 2 has buses 2, 4 and 5 with generator at bus- 4 . In the entire grid, bus -4 is considered as slack bus to meet the energy imbalance or transmission losses. The system bus data and line data are given in Appendix A. The generator units are treated as Gencos, load buses as Discos and the all transmission lines as single Transco entity in the market environment. With single-sided auction mechanism, the market will settle generation schedules for a pre-defined load. By the consideration of equal bidding parameters, the energy is balanced in the both areas and the network is simulated using PowerWorld simulator.

The AVR value for generator 1 is set at $1.05 \mathrm{pu}$ and for generator 2, it is $1.02 \mathrm{pu}$. From the base case simulation results as illustrated in Figure. 1, the transmission system is operating under normal state. The transmission system is subjected to $12.2 \mathrm{MW}$ real power loss and 24.4 MVAr reactive power loss. And as given in Table 2, all the bus voltages are within their satisfactory limits. Since generation and loads are balanced in the both areas, the amount of power exchange through tie-lines is only 4.75 MW from Area2 to Area- 1 towards transmission losses. The dispatch results are given in Table- 2 . 
Table 2. Results of base Case Simulation

\begin{tabular}{ccccccc}
\hline \hline \multirow{2}{*}{ Bus \# } & \multirow{2}{*}{ Area \# } & \multirow{2}{*}{ Voltage (p.u.) } & \multicolumn{2}{c}{ Generation } & \multicolumn{2}{c}{ Load } \\
\cline { 4 - 7 } & & & MW & MVAr & MW & MVAr \\
\hline 1 & 1 & 1.0500 & 100 & 104.95 & - & - \\
2 & 2 & 0.9562 & - & - & 50 & 35 \\
3 & 1 & 0.9599 & - & - & 50 & 35 \\
4 & 2 & 1.0200 & 112.21 & 59.46 & - & - \\
5 & 2 & 0.9220 & - & - & 50 & 35 \\
6 & 1 & 0.9404 & - & - & 50 & 35 \\
\hline \multicolumn{2}{r}{} & & 212.20 & 164.4 & 200 & 140 \\
\hline
\end{tabular}

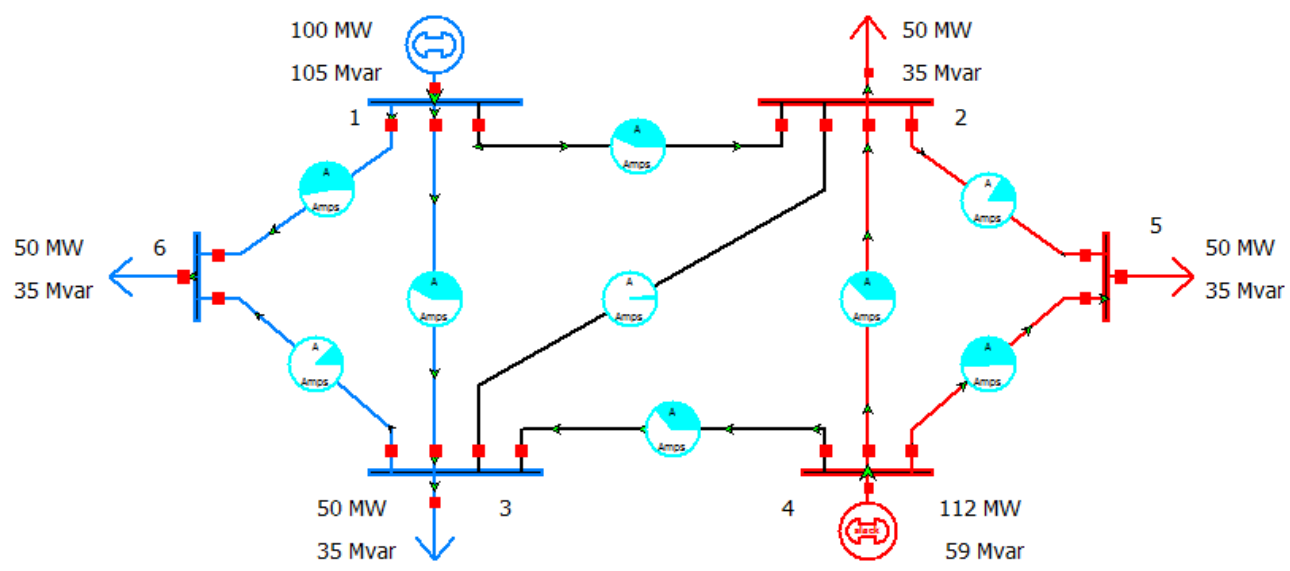

Figure 1. Simulation of Network for base Case Market Schedule

\subsection{Generators AVR Control}

\subsubsection{Case Study -1}

From the base case simulation, the system is not subjected to congestion. Hence, the system operator goal is to control transmission losses since allocation of transmission losses to the market participants is one of the important issues in the current environment. One of the important hidden factors is that high reactive power generation is happened with high AVR value for generator at bus-1 when compared with generator at bus -4 . Similarly, when AVR values for both generators are altered, then the reactive power generations are also changed accordingly as given in Table 3 . In addition to this, Area-1 has high voltage profile when compared with Area-2 due to high AVR value for generator at bus- 1 and the situation is quite reverse when generator at bus -4 with high AVR value in Area-2. This is clearly indicating that the system voltage profile directly impacts due to control with generator bus voltages. Hence it is worthwhile to optimize the generator bus voltages with an objective and our work is considered loss minimization as an objective function.

\subsubsection{Case Study -2}

In this case study, the entire load is planned to meet with generation in Area-2 with an assumption of high cost bidding scenario in Area-1. Since in competitive energy market, the participants can change their bidding strategy at any trading interval, the simulation is 
done with zero real power generation at bus-1. As we can see from the simulation results illustrated in Fig. 2, the reactive power generation is happening at bus-1 due to its on-line condition. Under this condition, the reactive power generation at bus- 1 can be considered as ancillary service for reactive power and voltage control from generation sources. We can able to see from Table 4 and Table 5, the high reactive power generation at bus 1 when it has high AVR value and decreased reactive power generation with low AVR value respectively. In other words, it is required to have sufficient reactive reserve for maintain good voltage profile in the network.

Table 3. Results with High AVR value for Generator at Bus 4

\begin{tabular}{ccccccc}
\hline \hline \multirow{2}{*}{ Bus \# } & \multirow{2}{*}{ Area \# } & \multirow{2}{*}{ Voltage (p.u.) } & \multicolumn{2}{c}{ Generation } & \multicolumn{2}{c}{ Load } \\
\cline { 4 - 7 } & 1 & 1.0200 & 100 & 64.60 & & \\
\hline 1 & 2 & 0.9600 & & & 50 & 35 \\
2 & 1 & 0.9563 & & & 50 & 35 \\
3 & 2 & 1.0500 & 111.97 & 99.34 & & \\
4 & 2 & 0.9404 & & & 50 & 35 \\
5 & 1 & 0.9222 & & & 50 & 35 \\
6 & Total & & 211.97 & 163.9 & 200 & 140 \\
\hline
\end{tabular}

Table 4. Generator 1 Real Power Output Zero at bus 1and High AVR Value

\begin{tabular}{|c|c|c|c|c|c|c|}
\hline \multirow{2}{*}{ Bus \# } & \multirow{2}{*}{ Area \# } & \multirow{2}{*}{ Voltage (p.u.) } & \multicolumn{2}{|c|}{ Generation } & \multicolumn{2}{|c|}{ Load } \\
\hline & & & MW & MVAr & MW & MVAr \\
\hline 1 & 1 & 1.0500 & 0 & 169.8 & - & - \\
\hline 2 & 2 & 0.9475 & - & - & 50 & 35 \\
\hline 3 & 1 & 0.9510 & - & - & 50 & 35 \\
\hline 4 & 2 & 1.0200 & 229.02 & 28.26 & - & - \\
\hline 5 & 2 & 0.9145 & - & - & 50 & 35 \\
\hline 6 & 1 & 0.9351 & - & - & 50 & 35 \\
\hline \multicolumn{3}{|c|}{ Total } & 229.02 & 198.16 & 200 & 140 \\
\hline
\end{tabular}

Table 5. Generator 1 Real Power Output Zero at Bus 1and Low AVR Value

\begin{tabular}{ccccccc}
\hline \multirow{2}{*}{ Bus \# } & \multirow{2}{*}{ Area \# } & \multirow{2}{*}{ Voltage (p.u.) } & \multicolumn{2}{c}{ Generation } & \multicolumn{2}{c}{ Load } \\
\cline { 4 - 7 } & 1 & 1.0200 & 100 & 64.60 & & \\
\hline 1 & 2 & 0.9600 & & & 50 & 35 \\
2 & 1 & 0.9563 & & & 50 & 35 \\
3 & 2 & 1.0500 & 111.97 & 99.34 & & \\
4 & 2 & 0.9404 & & & 50 & 35 \\
5 & 1 & 0.9222 & & & 50 & 35 \\
6 & Total & & 211.97 & 163.9 & 200 & 140 \\
\hline
\end{tabular}


The power interchange through tie-lines from Area-2 to Area-1 is about 106.72 MW including transmission losses in Area-1. By observing transmission line flows, line 4-3 is loaded around $86 \%$ to its rated MVA. Hence it is worthwhile to know the available transfer capability (ATC) between areas.

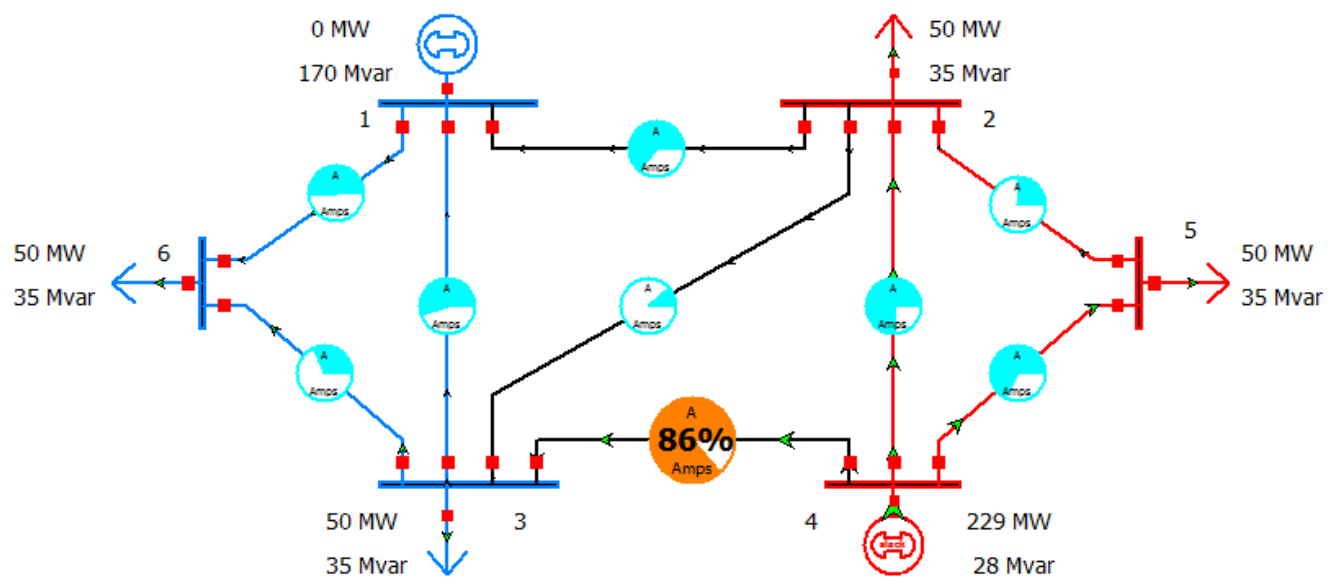

Figure 2. Simulation of Market Dispatch when Area-1 with High Price Bidding Strategy

\subsection{Load Curtailment}

The objective of this case study is to show the effectiveness of lack of reactive power support in the network. This study is extension to the case study 2 in which generator of Area-1 is outaged and the required load curtailment to overcome congestion problem is determined. A fictitious load is connected and with a negative sign the load is increased. For 44 MW load decrement in Area-1 causes to elevate congestion in the network as illustrated in Fig. 3. The same is repeated with high-AVR value at generator in Area-2 and for this case, the required load curtailment is only $30 \mathrm{MW}$ as illustrated in Fig. 4. Hence we can conclude that the proper voltage level with reactive power support can causes to less load curtailment during alert state in the network.

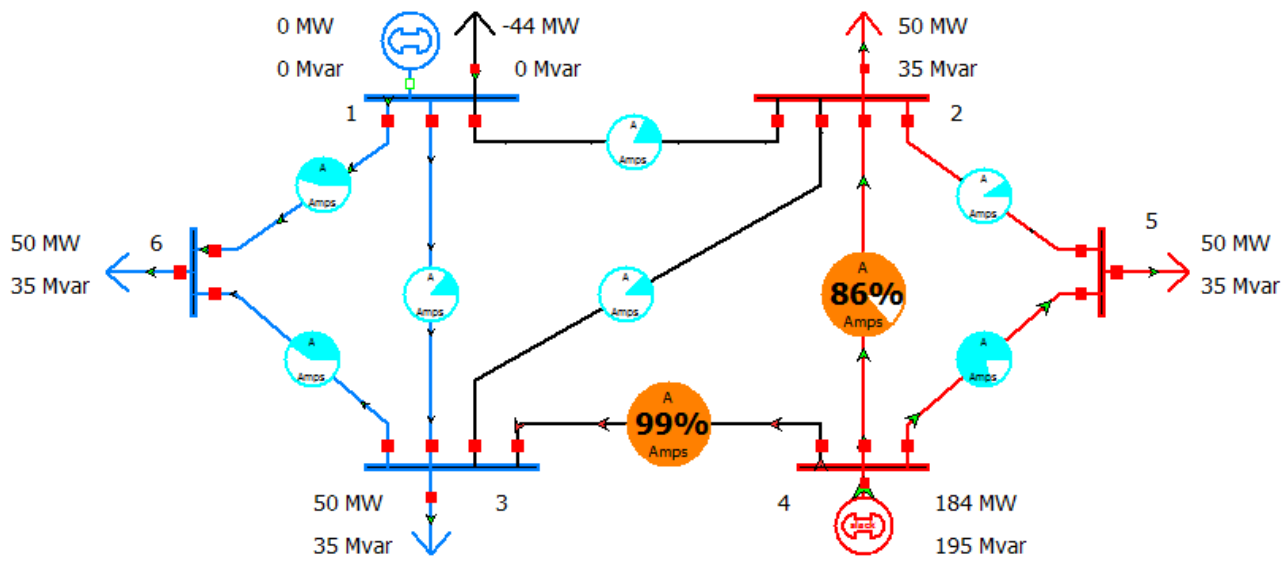

Figure 3. Load Curtailment with Low AVR Value for Generator at Bus 4 


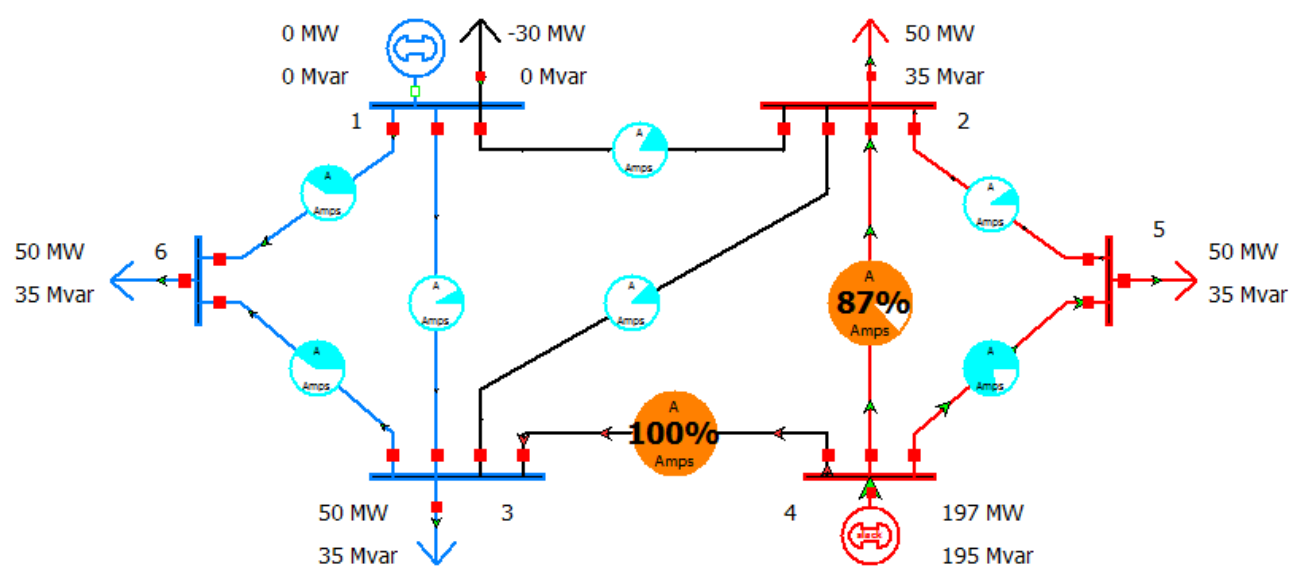

Figure 4. Load Curtailment with High AVR Value for Generator at Bus 4

\subsection{Control of Volt/VAr Supporting Devices}

\subsubsection{Case Study -1}

A forced outage of generator is created in the network. Under this situation, two of the transmission lines are violated their maximum thermal limit as shown in Fig. 5. A shunt capacitor is connected at bus 3 and its value is adjusted to $33.9 \mathrm{MVAr}$ and consequently the situation of congestion relief can visualize as shown in Fig. 6.

\subsubsection{Case Study -2}

Similarly, we have trailed a tap-changing transformer control in line 3-4. For a value of tap-setting at 1.02 p.u, the congestion is relieved in the network as shown in Fig. 7. The VAr control in the network is not only impacted on congestion but also reduced the transmission losses as well improved the bus voltage profile significantly. This case study is clearly indicating the need of Volt/VAr supporting devices control to manage congestion.

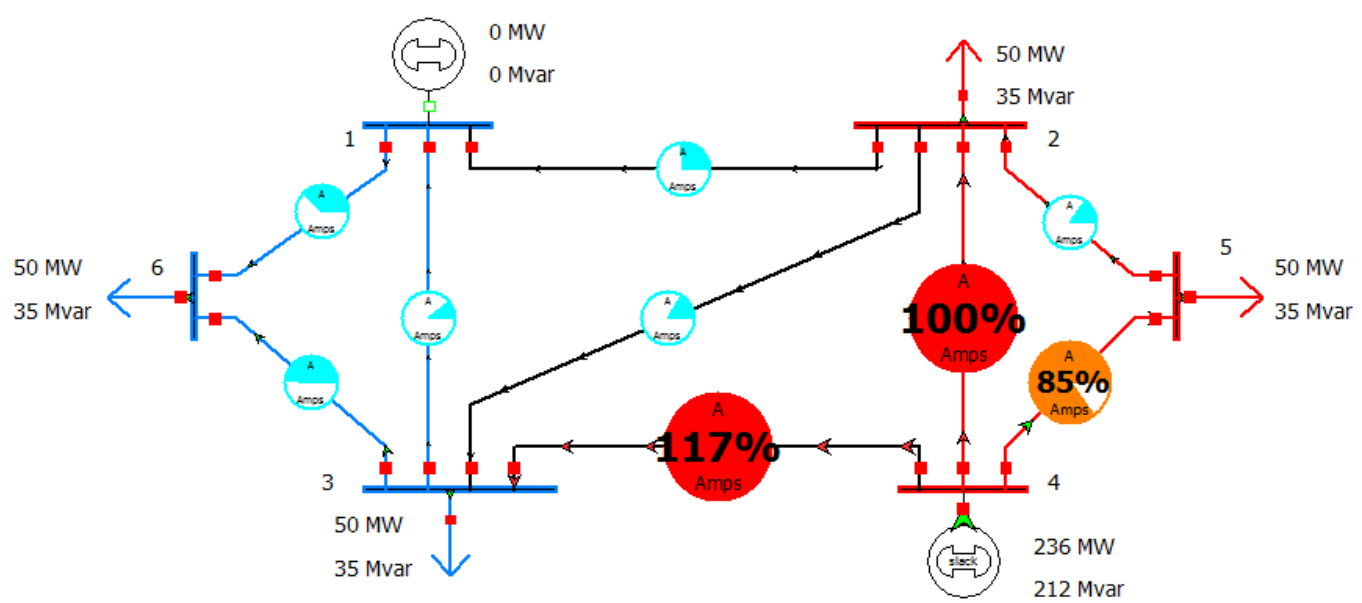

Figure 5. Congestion State under Generator Outage 


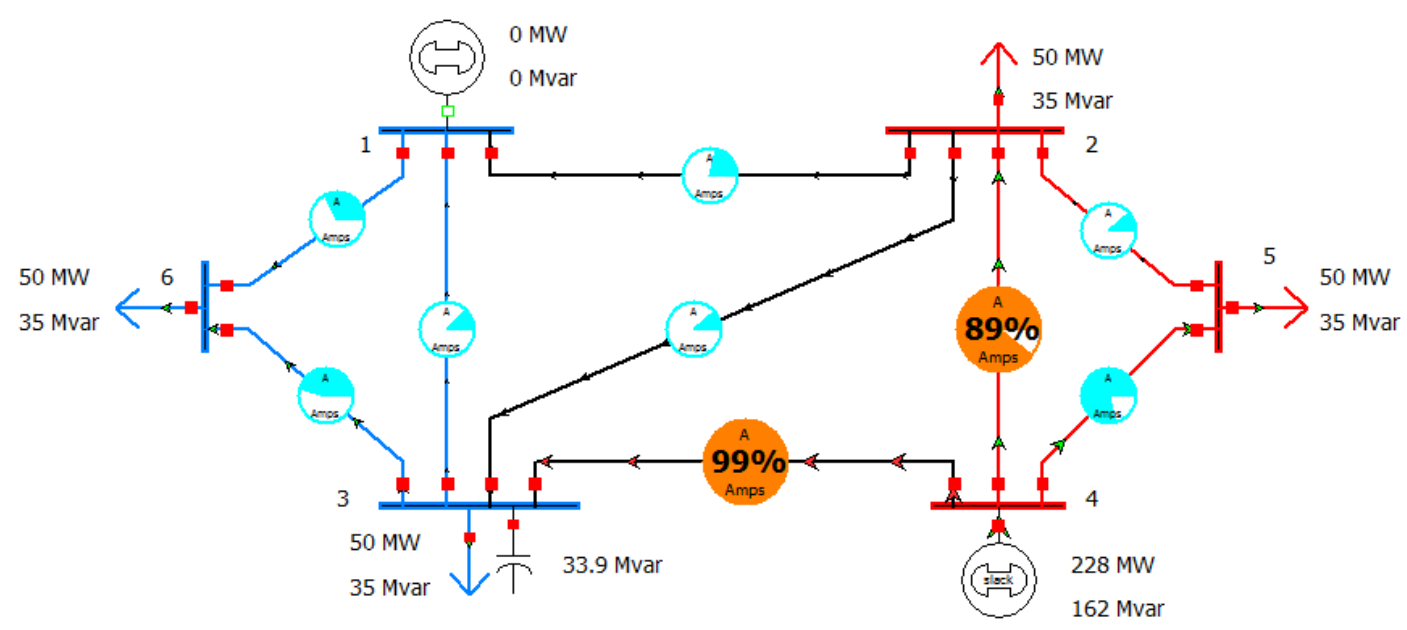

Figure 6. Simulation of Shunt VAr Control and its Impact on Congestion

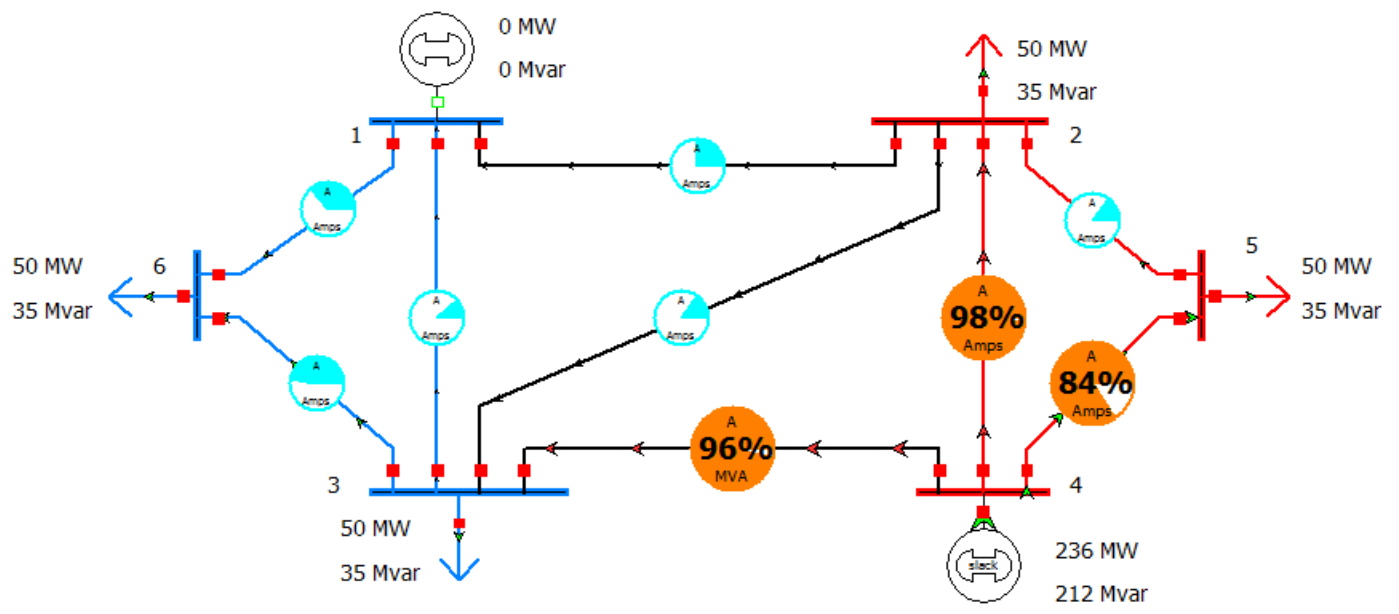

Figure 7. Simulation of Tap-changing Transformer Control and its Impact on Congestion

\subsection{Operation of FACTS Devices}

Regardless of expansion strategies constrained by technical and economical aspects, the maximum utilization of transmission system is become essential to the system operators in many grids around the world. The improvement of transmission technologies result in an efficient grid operated by the system operators. Power controlling devices such as Flexible AC Transmission Systems (FACTS) which emerged in 1980s are redefined the entire preventive/control actions for system security and reliability enhancement. Many of the researchers are already focused on these devices to mitigate intolerable operating states for security maintenance. In order to have all desired benefits from FACTS devices, their location and parameters optimization plays a key role.

\subsubsection{Case Study -1}

In the present case study, line 3-4 is heavily congested and the equivalent of decoupled power injection modeling of UPFC has been incorporated in the same line. The power injection at bus 3 and withdraw at bus 4 is made equal to zero for the absence of UPFC operation at initial case as shown in Fig. 8. 


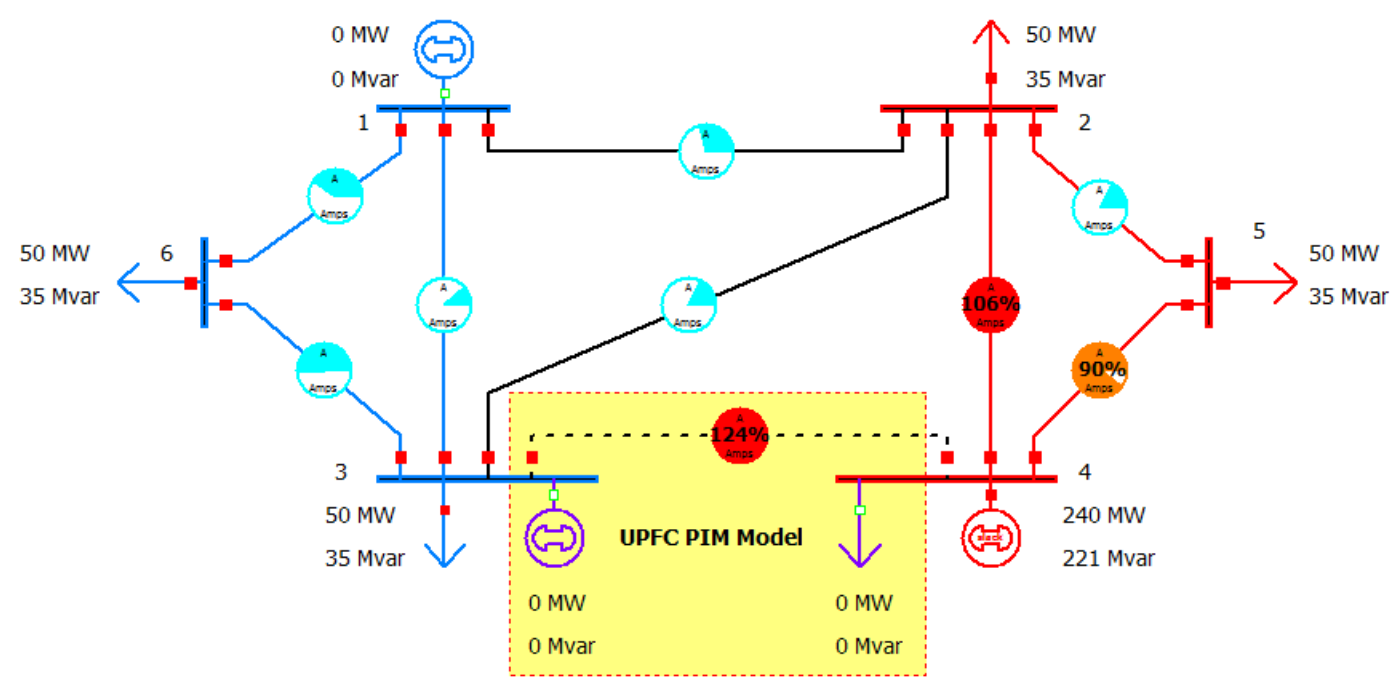

Figure 8. Integration of DPIM Equivalent of UPFC in Line 3-4

According to decoupled power injection modeling (DPIM) of UPFC [4], the required power balance at bus 3 and bus 4 is made equal to power flow in line $3-4$ as shown in Fig. 3. We can have the situation of congestion relief as shown in Fig. 9.

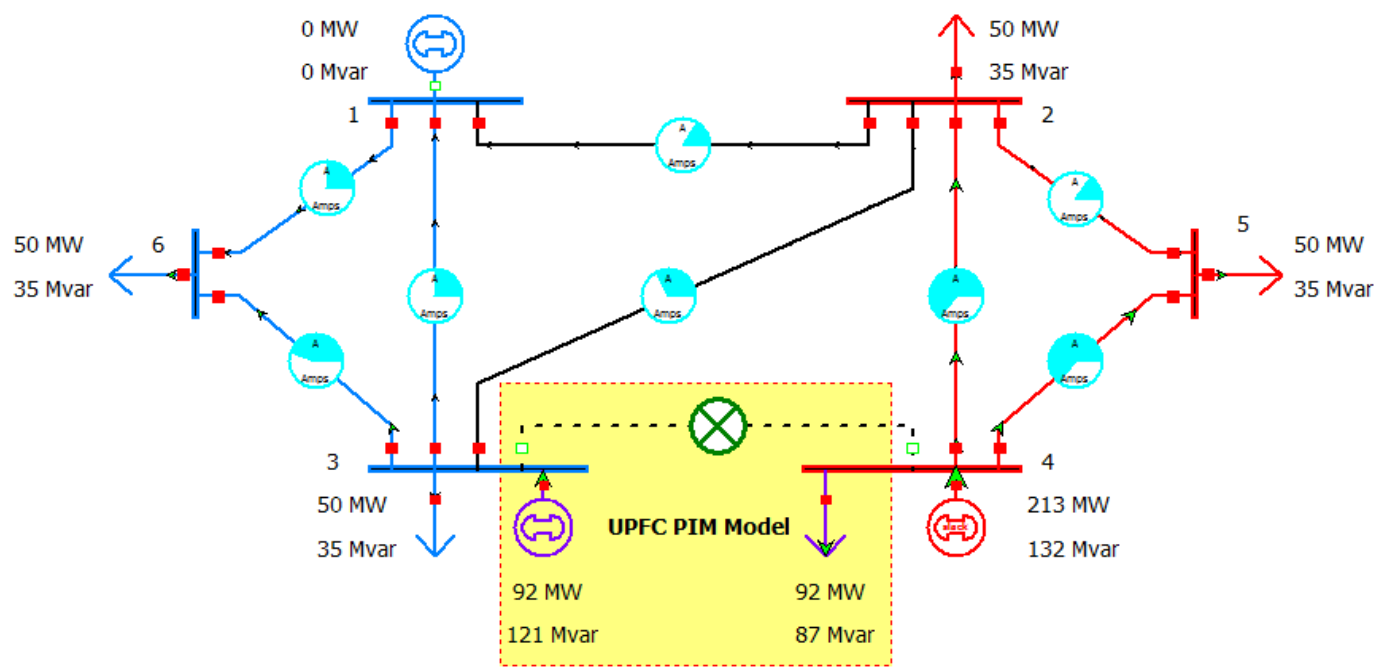

Figure 9. DPIM and UPFC Operation in Line $3-4$

\subsubsection{Case Study -2}

In order to explore UPFC benefits apart from congestion relief we have estimated the available transfer capability (ATC) [5] from Area 2 to Area 1. A fictitious load is added at bus 1 and it value is adjusted up to one of the transmission line reaches to its MVA rating. For $42 \mathrm{MW}$ load at bus 1, line $2-4$ reaches to $99 \%$ of its MVA as shown in Figure. 10. The similar exercise is done with high AVR value for generator at bus 4 and considerably ATC is increased from $42 \mathrm{MW}$ to $46 \mathrm{MW}$ as shown in Figure. 11. It means, by the proper control of all Volt/VAr supporting devices and UPFC simultaneously, the system security margin can increase significantly. 


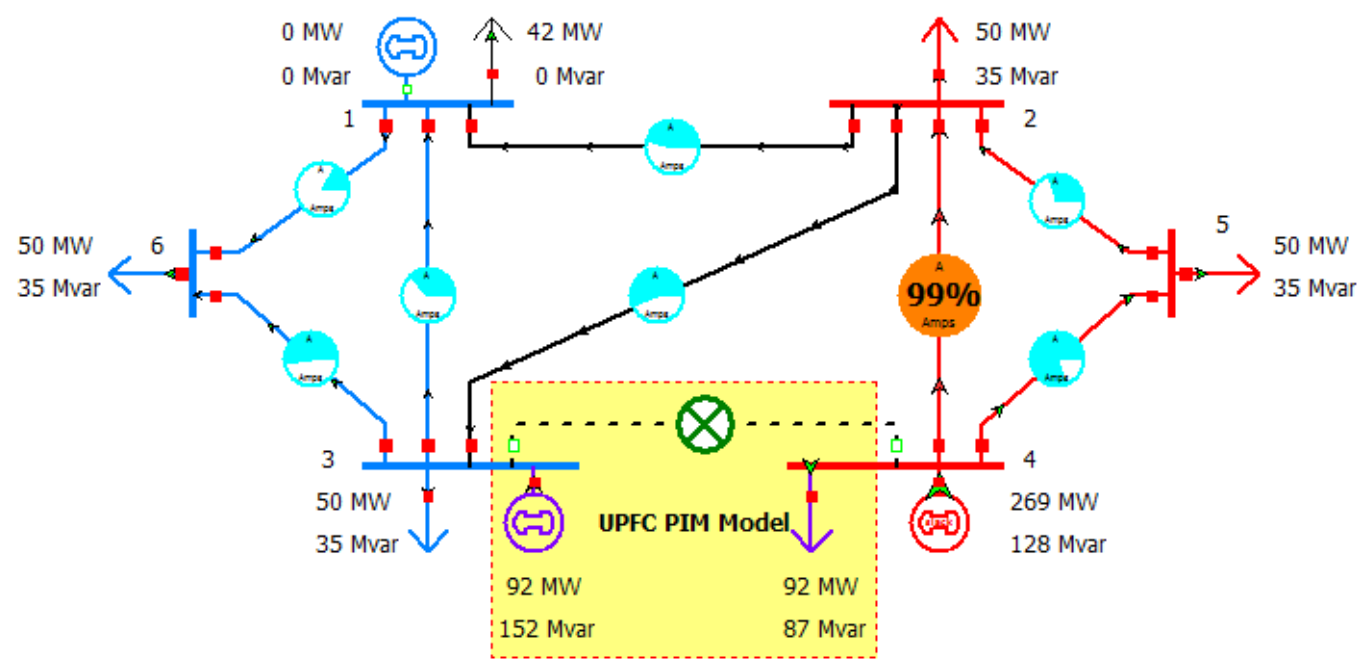

Figure 10. ATC Calculation with UPFC and low AVR Value at Bus 4

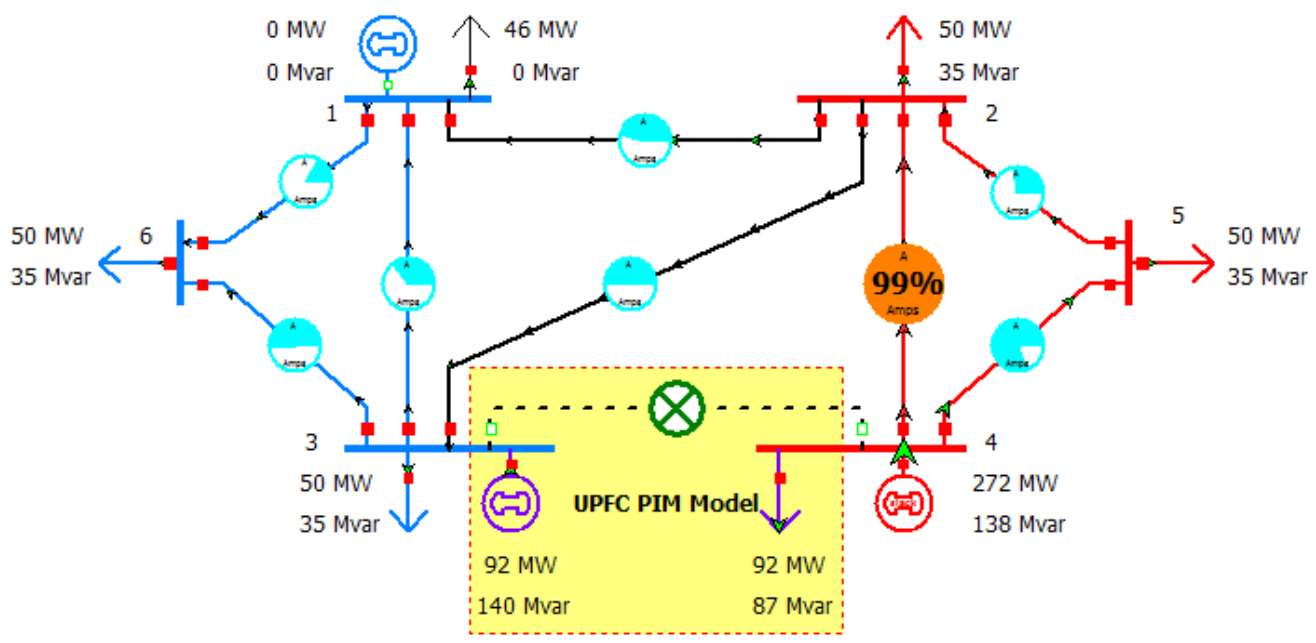

Figure 11. ATC Calculation with UPFC and High AVR Value at Bus 4

\section{Conclusion}

In this paper, we have illustrated various congestion relief approaches which can significantly avoid market economic inefficiency. The load curtailment is not preferable in social welfare point of view, but it is required to control the network under inevitable situation. Under congestion, the redispatch can causes to increase generation cost. To avoid this, preventive measures like integration of FACTS and Volt/VAr controlling devices in network is essential. The impact of any congestion relief approach on market economics has to understand and will be the extension of this work. 


\section{References}

[1] A. Kumar, S. C. Srivastava and S. N. Singh, "Congestion management in competitive power market: A bibliographical survey", Electric Power Systems Research, vol. 76, no. 1-3, (2005), pp. 153-164.

[2] K. R. S. Reddy, N. P. Padhy and R. N. Patel, "Congestion management in deregulated power system using FACTS devices", Proceedings of IEEE Power India Conference, (2006); New Delhi. India.

[3] D. Das and B. F. Wollenberg, "Risk Assessment of Generators Bidding in Day-Ahead Market", IEEE Trans. on Power Systems", vol. 20, no. 1, (2005), pp. 416-424.

[4] S. A. Nabavi-Niaki and M. R. Iravani, "Steady State and Dynamic Models of Unified Power Flow Controller (UPFC) for Power System Studies", IEEE trans. on power systems, vol. 11, no. 4, (1996), pp. 1937-19436.

[5] G. C. Ejebe, J. Tong, J. G. Waight, J. G. Frame, X. Wang and W. F. Tinney, "Available transfer capability calculations", IEEE Transactions on Power Systems , vol.13, no. 4, (1998), pp. 1521,1527. 